\title{
Characterization of zinc transporter 8 (ZnT8) antibodies in autoimmune diabetic patients from Argentinian population using monomeric, homodimeric, and heterodimeric ZnT8 antigen variants
}

\author{
Natalia I Faccinetti, Luciano L Guerra, Alberto Penas Steinhardt, Ruben F lacono,

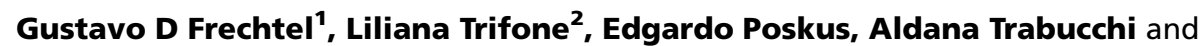 \\ Silvina N Valdez ${ }^{+}$
}

Chair of Immunology, School of Pharmacy and Biochemistry, University of Buenos Aires (UBA), and 'Prof. Ricardo A. Margni' Humoral Immunity Studies Institute (IDEHU), National Research Council (CONICET)-UBA, Ciudad Autónoma de Buenos Aires, Argentina, 'Genetic Division, Clinical Hospital, UBA, Genetic, Immunology, Metabolism Institute (INIGEM), CONICET-UBA, Ciudad Autónoma de Buenos Aires, Argentina and ${ }^{2}$ Diabetes and Nutrition Service, National Pediatric Hospital 'Dr. Ricardo Gutierrez', Ciudad Autónoma de Buenos Aires, Argentina

${ }^{\dagger}$ (S N Valdez is now at Cátedra de Inmunología, Facultad de Farmacia y Bioquímica, Universidad de Buenos Aires, Junín 956, $4^{\circ}$ piso, C1113AAD Ciudad Autónoma de Buenos Aires, Argentina)

\author{
Correspondence \\ should be addressed \\ to $\mathrm{S} N$ Valdez \\ Email \\ silval@ffyb.uba.ar
}

\begin{abstract}
Objective: In order to gain further knowledge of the structure of zinc transporter 8 (ZnT8) epitopes, we studied the role of the amino acid at position 325 in the antigen and its dimeric conformation for autoantibodies to ZnT8 (ZnT8A) recognition. Methods: For this purpose, several ZnT8 C-terminal domain variants were designed: monomer carrying Arg325 or Trp325, homo-dimers ZnT8-Arg-Arg325 and ZnT8-Trp-Trp325, and hetero-dimer ZnT8-Arg-Trp325. Two groups of Argentinian diabetic patients were subjected to analysis using $\left.{ }^{35} \mathrm{~S}\right]-\mathrm{ZnT}$ (8 variants by radioligand binding assay (RBA): i) 100 new-onset, insulin-dependent, type 1 diabetic patients and ii) 282 slowly progressing to insulin requirement, non-obese adult-onset diabetic patients. In addition, 50 type 1 diabetic patients and 100 normal control sera provided by the American Diabetes Association (ADA) were evaluated in order to calculate the sensitivity and specificity of ZnT8A assays for each antigenic variant. Other routine $\beta$-cell autoantibodies were also tested by RBA.

Results: Of the 100 Argentinian type 1 diabetic patients, 65 were ZnT8A+. Out of them, 8 patients recognized all recombinant forms of ZnT8 and most patients (56) reacted against the heterodimer. Additionally, out of 282 non-obese adultonset diabetic patients 46 were ZnT8A+, whereas 29 patients recognized only dimers. Besides, exclusive reactivity against ZnT8A was found in $9.0 \%$ for type 1 diabetes mellitus and $10.3 \%$ for non-obese adult-onset diabetic patients. Conclusions: Significantly higher signal values in RBA were obtained with the heterodimeric variant. An increased detection of humoral autoimmunity was found in both groups when ZnT8A was employed in combination with the other $\beta$-cell autoantibodies. The inclusion of homodimeric immunoreactive peptides revealed the existence of quaternary structuredefined epitopes probably resembling the actual state of the autoantigen in vivo. Finally, the differential profiles of ZnT8A exhibited by type 1 and non-obese adult-onset diabetic patients suggest the different nature of autoimmune processes underlying both pathologies.
\end{abstract}




\section{Introduction}

Zinc transporter 8 (ZnT8) is a multipass transmembrane protein which has been identified as a novel autoantigen in patients with type 1 diabetes mellitus (DM) (1). It is a 369 amino acid protein, encoded by the SLC30A8 gene located in the chromosome 8q24.11. ZnT8 transports zinc ions from the $\beta$ cell cytoplasm into insulin-secretory vesicles where they are essential for the proper storage and secretion of insulin (2). The expression of ZnT8 is remarkably restricted, being almost exclusively confined to pancreatic $\beta$ cells (3). In a murine model, Murgia et al. (4) have demonstrated that ZnT8 is localized, at far lower levels, in pancreatic $\alpha$ cells, epithelial cells within thyroid follicles, and in the adrenal cortex. The immunoreactivity against ZnT8 within the islet is found both in $\beta$ and $\alpha$ cells, which is surprising since glucagon storage is not known to require zinc $(5,6,7)$.

Genome-wide association studies analyzing susceptible/ protective loci for type $2 \mathrm{DM}$ revealed that a nonsynonymous single nucleotide polymorphism (SNP) in SLC30A8 (rs13266634 C > T) is associated with the disease (8). This SNP changes arginine (Arg) to tryptophan (Trp) at position 325, with a higher risk of developing the disease when the allele $\mathrm{C}(\mathrm{Arg} 325)$ is present.

Among the major autoantibodies employed in the diagnosis of autoimmune DM, autoantibodies to ZnT8 (ZnT8A) are the most recently described humoral marker, complementing those already used such as insulin/ proinsulin autoantibodies (IAA/PAA), glutamic acid decarboxylase autoantibodies (GADA), and insulinoma associated protein tyrosine phosphatase 2 autoantibodies (IA-2A). ZnT8A have been detected in more than $60 \%$ of patients with type $1 \mathrm{DM}(9,10)$ and in more than $10 \%$ of adult-onset diabetic patients (11).

Wenzlau et al. and other authors have reported that ZnT8-reactive sera mostly recognize the C-terminal domain of the molecule (amino acids 268-369), where the variant residue at amino acid 325 is located $(1,10)$. Previous studies have demonstrated that ZnT8A displays specific immunoreactivities against the hybrid dimeric construction ZnT8-Arg-Trp325, although they do not recognize ZnT8-Arg325 nor ZnT8-Trp325 (monomeric antigen constructions) (10). This observation suggests that conformational non-continuous epitopes present in the ZnT8 dimeric construction are detected by ZnT8A, emulating the mechanism of immune response triggered by the quaternary structure of this autoantigen in vivo (4).

In a previous work, we have characterized ZnT8A prevalence in Argentinian non-obese adult-onset diabetic patients only using monomer and hetero-dimer variants of the ZnT8 C-terminal domain (11). In the present study, the role of amino acid at position 325 and dimeric construction were analyzed in order to gain further knowledge of the structure of ZnT8 epitopes and ZnT8A recognition. For this purpose, we also included homodimers ZnT8 variants and two groups of Argentinian diabetic patients underwent analysis: i) new-onset, insulin dependent, type 1 diabetic patients and ii) slowly progressing to insulin requirement, non-obese adultonset diabetic patients.

\section{Subjects and methods}

\section{Sera from Diabetes Antibody Standardization Program}

One hundred and fifty sera, provided by the American Diabetes Association (ADA) to participate in the Diabetes Antibody Standardization Program (DASP 2009), were evaluated in order to calculate the sensitivity and specificity of ZnT8A assays. These samples included 50 sera from new-onset type 1 diabetic patients (17 females and 33 males, median age of 24.5 years (range 10-32 years), 48 Caucasians, 1 African-American, and 1 Asian) (12). Furthermore, 100 normal control sera were used to calculate the specificity.

\section{Argentinian population}

Normal human controls $>$ Normal human control sera $(n=50)$ were obtained from healthy subjects without personal or family history of diabetes or autoimmune disease. These sera were used to determine the cut-off value of each assay. The collection of serum samples was approved by the Ethics Committee of the Clinical Hospital José de San Martín, University of Buenos Aires (UBA), Buenos Aires, Argentina. All subjects were informed about the purpose of the study, and a signed consent for study participation was obtained.

Type 1 diabetic patients $>$ This group included 100 children and adolescents admitted to the Nutrition Service at Gutierrez National Pediatric Hospital (Buenos Aires, Argentina) from May 2013 to March 2015. Clinical and laboratory data for these patients are given in Supplementary Table 1, see section on supplementary data given at the end of this article. As the hospital is a referral center, patients came from all over Argentina and 
were mainly Caucasians. Serum samples were collected before or within $72 \mathrm{~h}$ of starting insulin treatment. Type 1 DM was diagnosed according to WHO criteria (13).

Non-obese adult-onset diabetic patients $>$ A population of 282 subjects attending the Division of Diabetes at the Clinical Hospital José de San Martín, from 2002 to 2004, who had been diagnosed with DM at the age $>30$ years (mean age of 53.4 years, range $30-84$ years), with a BMI $\leq 30(24.6 \pm 2.9)$, without insulin treatment for the first year of disease, and initially classified as type 2 diabetic patients, were included in this study. Diagnosis was performed according to the ADA criteria (14). The patients included in this study had not been treated with insulin before blood sample collection for immunochemical analysis. Relevant clinical information is shown as Supplementary Table 2, see section on supplementary data given at the end of this article.

Sample collection - Blood samples were collected after overnight fasting and sera were stored at $-20^{\circ} \mathrm{C}$ until assayed. Sera collection from newly diagnosed type 1 diabetic patients and adult-onset diabetic patients, and the respective protocols were approved by the Ethical Committees of the Gutierrez National Pediatric Hospital and Clinical Hospital José de San Martín respectively. Written consent from all participants involved in this study, and parental consent when being a minor, were obtained.

Statement of human rights $>$ All procedures followed were in accordance with the ethical standards of the responsible committee on human experimentation (Clinical Hospital José de San Martín, University of Buenos Aires (UBA), and Gutierrez National Pediatric Hospital, Buenos Aires, Argentina) and with the Helsinki Declaration of 1975, as revised in 2008 (27).

\section{DNA constructs}

The cDNA plasmids encoding the C-terminal domains of ZnT8 (amino acids 268-369) carrying Arg325 or Trp325 were kindly provided by Dr J Hutton from the Barbara Davis Center of Childhood Diabetes, University of Colorado, USA. In addition, three dimeric cDNA constructs carrying Arg325-Arg325, Trp325-Trp325, and Arg325Trp325 were synthesized by GenScript (Piscataway, NJ, USA) and ligated into the PstI/XbaI sites of p-ALTER-Ex1 plasmid (Promega). The linker peptide used to generate the dimers was PKPSTPPGSSGGGS. The identity of the new DNA molecules was corroborated by sequencing.

\section{Radioligand binding assay protocol for ZnT8A}

ZnT8A were assessed by radioligand binding assay (RBA) as previously described (11). Briefly, cDNA coding for ZnT8Arg325, ZnT8-Trp325, ZnT8-Arg-Arg325, ZnT8-TrpTrp325, and ZnT8-Arg-Trp325 were transcribed and translated using a rabbit reticulocyte lysate system (Promega Corporation) and $\left[{ }^{35} \mathrm{~S}\right]$-methionine (New England Nuclear, Boston, MA, USA) according to manufacturer's instructions. The RBA was carried out by incubating $5 \mu \mathrm{l}$ of human sera overnight at $4{ }^{\circ} \mathrm{C}$ with 10000 c.p.m. of the $\left[{ }^{35} \mathrm{~S}\right]-Z n T 8-A r g 325,\left[{ }^{35} \mathrm{~S}\right]-Z n T 8-T r p 325,\left[{ }^{35} \mathrm{~S}\right]-Z n T 8-$ Arg-Arg325, [ $\left.{ }^{35} \mathrm{~S}\right]-Z n T 8-T r p-T r p 325$, or $\left[{ }^{35} \mathrm{~S}\right]-Z n T 8-A r g-$ Trp325 in a final volume of $60 \mu \mathrm{l}$ in RBA buffer $(0.02 \mathrm{M}$ Tris-HCl, $0.15 \mathrm{M} \mathrm{NaCl}, 0.15 \%$ Tween 20, pH 7.4), $0.1 \%$ aprotinin, and $0.1 \%$ BSA.

Subsequently, isolation of immune complexes was carried out by addition of $50 \mu 150 \%$ protein A-Sepharose 4B FF (GE, Healthcare BioScience, Upsala, Sweden) in RBA buffer. Pellets were washed three times with $200 \mu \mathrm{l}$ RBA buffer and once with $200 \mu 10.20 \mathrm{M} \mathrm{NaCl}$ in RBA buffer. Finally, pellets were suspended in $100 \mu \mathrm{l} 1 \%$ sodium dodecyl sulphate and supernatants were carefully transferred to vials for scintillation counting. Results were expressed as $\mathrm{B} \%=100 \times$ (bound c.p.m./total c.p.m.) and also as precision units, s.D.s. $=(\mathrm{B} \%-\mathrm{BC} \%) /$ s.D.C, where S.D.s. is standard deviation scores, $\mathrm{BC} \%$ is the control mean B\% and S.D.C its S.D. Thirty normal control sera were included in each assay demonstrating that $\mathrm{BC} \%$ was normally distributed. The selection of optimal cut-off values was based on curves constructed by plotting the calculated specificity and sensitivity of each assay against the corresponding cut-off values ranging from 1 to 6 s.D.s. The highest sensitivity combined with the highest specificity was observed at a cut-off value of 3 S.D.s. for each assay. The inter-assay coefficient of variation $(n=3)$ were $31.2,19.8,15.4,21.5$, and 10.3 for s.D.s. of 37.3, 23.5, 22.5, 6.8, and 10.3 for ZnT8-Arg325, ZnT8Trp325, ZnT8- Arg-Arg325, ZnT8-Trp-Trp325, and ZnT8Arg-Trp325 respectively.

\section{Detection of other anti-islet autoantibodies}

GADA and IA-2A were determined by RBA as previously described (15). In brief, cDNA coding for human GAD65 or tyrosine-phosphatase IA-2 was transcribed and translated using a rabbit reticulocyte lysate system in the presence of $\left[{ }^{35} \mathrm{~S}\right]$-methionine as indicated for the ZnT8A assay. The RBA was carried out by incubating $2.5 \mu \mathrm{l}$ of human sera overnight at $4{ }^{\circ} \mathrm{C}$ with 10000 c.p.m. 
of $\left[{ }^{35} \mathrm{~S}\right]$-GAD or $\left[{ }^{35} \mathrm{~S}\right]-\mathrm{IA}-2$ in a final volume of $60 \mu \mathrm{l}$ in RBA buffer.

Subsequently, the isolation of immune complexes and washing steps were done as described for the ZnT8A assay. Results were expressed as S.D.s. In the DASP 2007, our GADA and IA-2A assays proved to have sensitivities of 80 and $69 \%$ and specificities of 98 and $97 \%$ respectively. PAA was also determined by RBA as described by Valdez et al. (16). The cDNA coding for human proinsulin (PI) was transcribed and translated using a rabbit reticulocyte lysate system in the presence of $\left[{ }^{35} \mathrm{~S}\right]$-cysteine (New England Nuclear), according to the manufacturer's instructions. After overnight refolding, accomplished by a disulphide reduction-reoxidation procedure, $\left[{ }^{35} \mathrm{~S}\right]$-PI was isolated by reverse-phase HPLC. Sera $(30 \mu \mathrm{l})$ were incubated for 7 days at $4{ }^{\circ} \mathrm{C}$ with 1000 c.p.m. of $\left[{ }^{35} \mathrm{~S}\right]-\mathrm{PI}$ in $90 \mu \mathrm{l}$ of RBA buffer. Subsequently, isolation of immune complexes and wash steps were done as indicated above. Results were expressed as s.D.s.

\section{Statistical analysis}

The statistical analysis was performed using GraphPad Prism Software, version 6.0 for Windows (San Diego, CA, USA, www.graphpad.com). Results were expressed as mean \pm s.D. unless otherwise indicated. To assess if data from normal human control sera were normally distributed, the D'Agostino and Pearson omnibus normality test was applied. The Mann-Whitney $U$ test was used to evaluate significantly differences in S.D.s. from RBA-positive ZnT8A sera between ZnT8-Arg325, ZnT8-Trp325, ZnT8Arg-Arg325, ZnT8-Trp-Trp325, and ZnT8-Arg-Trp325 assays. Differences in non-parametric data were tested by the Mann-Whitney $U$ test. A $P$ value $<0.05$ was considered statistically significant.

\section{Results}

\section{Sensitivity and specificity of RBA employing ZnT8 variants}

In order to evaluate the sensitivity and specificity of RBA using the different ZnT8 probes, 150 sera from DASP 2009 were analyzed (Fig. 1). The sensitivity was calculated as the percentage of type 1 diabetic patients $(n=50)$ that scored positive by RBA using each variant. The specificity was calculated as 100 minus the percentage of normal human sera $(n=100)$ detected as positive by RBA using each variant of ZnT8.

Out of the 50 type 1 diabetic patients, 11 (22.0\%) had autoantibodies against ZnT8-Arg325, 13 (26.0\%) against

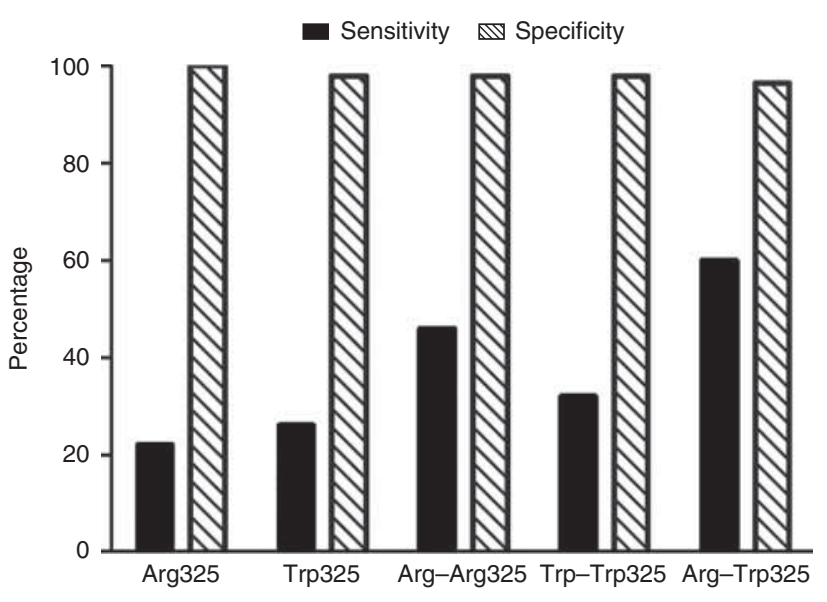

Figure 1

Sensitivity and specificity of RBA using each ZnT8 variant calculated with 150 sera provided by ADA. The sensitivity was calculated as percentage of results scored positive ( $n=50$ type 1 diabetic patients). Specificity was calculated as 100 minus the percentage of normal human sera detected as positive ( $n=100$ normal control).

ZnT8-Trp325, 23 (46.0\%) against ZnT8-Arg-Arg325, 16 (32.0\%) against ZnT8-Trp-Trp325, 30 (60.0\%) presented autoantibodies against the heterodimeric construct ZnT8-Arg-Trp325, and 19 (38.0\%) did not have autoantibodies against any ZnT8 variants. In addition, the specificity was $100 \%$ forZnT8-Arg325, 98\% for ZnT8-Trp325, ZnT8-Arg-Arg325, and ZnT8-TrpTrp325 and 96.5\% for ZnT8-Arg-Trp325.

\section{Prevalence of ZnT8A and association with other autoimmune markers}

One hundred Argentinian type 1 diabetic patients and 282 Argentinian non-obese adult-onset diabetic patients were tested in parallel for ZnT8A, PAA, GADA, and IA-2A.

Histogram in Fig. 2A shows the prevalence of ZnT8A in comparison with those from the other autoimmune markers in type 1 diabetic patients. ZnT8A were found in 65 out of 100 type 1 diabetic patients (65.0\%). The prevalence for ZnT8A was composited by reactivity to ZnT8-Arg325, ZnT8-Trp325, ZnT8-Arg-Arg325, ZnT8-TrpTrp325, and ZnT8-Arg-Trp325. The prevalence of the other autoantibodies in this group was $22.0 \%$ for PAA, $58.0 \%$ for GADA, and $53.0 \%$ for IA-2A.

A Venn's diagram in Fig. 2B illustrates the isolated and overlapping prevalence of PAA, GADA, IA-2A, and ZnT8A for this group of patients. It is noteworthy that nine 


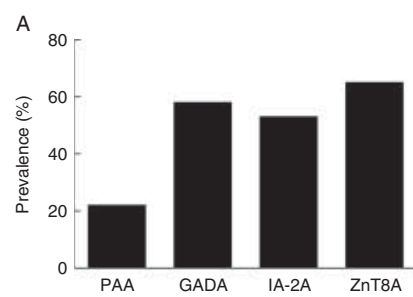

B

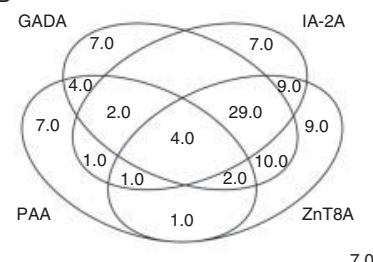

7.0
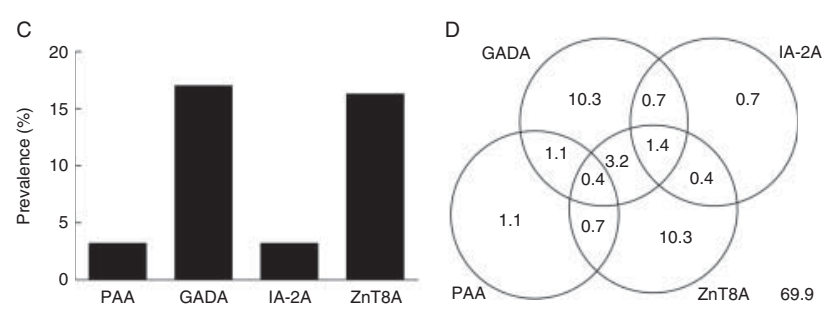

\section{Figure 2}

Prevalence ( $A$ and $C$ ) and marker profile (B and D) of ZnT8A in comparison with other autoimmune markers in Argentinian diabetic patients. Panels A and B: Type 1 diabetic patients ( $n=100)$; Panels C and D: non-obese adult-onset diabetic patients $(n=282)$. The prevalence for ZnT8A was composited by reactivity to ZnT8-Arg325, ZnT8-Trp325, ZnT8-Arg-Arg325,

ZnT8-Trp-Trp325, and ZnT8-Arg-Trp325. Results were expressed as percentages.

patients (9.0\%) were only positive for ZnT8A and the inclusion of this marker lowered the percentage of patients who tested negative in all four assays to $7.0 \%$.

On the other hand, out of 282 non-obese adult-onset diabetic patients, 9 (3.2\%) were PAA+, 47 (17.0\%) were GADA +, 9 (3.2\%) were IA-2A+, and 46 (16.3\%) were $\mathrm{ZnT} 8 \mathrm{~A}+$. As stated above, the prevalence for ZnT8A was composited by reactivity to all the constructs previously described (Fig. 2C). In addition, Fig. 2D illustrates the isolated and overlapping prevalence of PAA, GADA, IA-2A, and $\mathrm{ZnT} 8 \mathrm{~A}$ for this group of patients. In this case, 29 patients (10.3\%) were only positive for ZnT8A, increasing the detection of autoimmunity to $30.1 \%$.

We also analyzed the clinical characteristics of type 1 and non-obese adult-onset diabetic patients according to the presence of autoantibodies (Supplementary Table 3, see section on supplementary data given at the end of this article). In type $1 \mathrm{DM}$, the age at diagnosis was significantly lower in the group with at least one autoantibody than the one without any marker. On the other hand, in non-obese adult-onset diabetic patients there was only a significant difference between groups (with or without antibodies) when BMI was analyzed, being lower in the autoantibodies positive group.
This difference was also statistically significant $(P=0.001)$ after adjustment for covariates (age and sex).

\section{Prevalence of autoantibodies to each ZnT8 variants}

Table 1 depicts the percentage of $\mathrm{ZnT} 8 \mathrm{~A}+$ results by RBA using each variant in type 1 and non-obese adult-onset Argentinian diabetic patients. Out of the 100 type 1 diabetic patients, 42 had autoantibodies against ZnT8Arg325, ten against ZnT8-Trp325, 38 against ZnT8-ArgArg325, 15 against ZnT8-Trp-Trp325, and 56 presented autoantibodies against the heterodimeric construct ZnT8Arg-Trp325. On the other hand, out of the 282 non-obese adult-onset diabetic patients, two had autoantibodies against ZnT8-Arg325, 15 against ZnT8-Trp325, 23 against ZnT8-Arg-Arg325, nine against ZnT8-Trp-Trp325, and 18 against ZnT8-Arg-Trp325.

The signal values of ZnT8A for each antigenic variant were further compared in both groups of patients (Fig. 3 and Table 2). ZnT8A positive sera from type 1 diabetic patients had significantly higher RBA signal values when the heterodimeric variant was employed than those from RBAs using other antigenic probes: median 17.99 for ZnT8-Arg-Trp325 vs 10.71, 6.32, 5.66, and 4.44 s.D.s. for ZnT8-Arg325, ZnT8-Trp325, ZnT8-Arg-Arg325, and ZnT8Trp-Trp325, respectively; $P<0.05$, Mann-Whitney $U$ test.

For non-obese adult-onset diabetic patients, signals obtained with the heterodimer were also significantly higher than the ones corresponding to the remaining ZnT8 variants (median 12.87 for ZnT8-Arg-Trp325 vs 5.61, 5.78, and 6.07 s.D.s. for, ZnT8-Trp325, ZnT8-Arg-Arg325, and ZnT8-Trp-Trp325, respectively; $P<0.05$, Mann-Whitney $U$ test). Since only two samples were positive when ZnT8-Arg325 was used, no statistical comparison could be performed.

Table 1 Comparison of ZnT8A results obtained by RBAs using different antigenic variants on 100 sera from type 1 diabetic patients and 282 sera from non-obese adult-onset diabetic patients.

\begin{tabular}{|c|c|c|}
\hline & \multicolumn{2}{|c|}{ Prevalence $^{a}, n(\%)$} \\
\hline & $\begin{array}{c}\text { Type } 1 \text { DM } \\
(n=100)\end{array}$ & $\begin{array}{l}\text { Adult onset DM } \\
\qquad(n=282)\end{array}$ \\
\hline ZnT8-Arg325 & $42(42.0)$ & $2(0.7)$ \\
\hline ZnT8-Trp325 & $10(10.0)$ & $15(5.3)$ \\
\hline ZnT8-Arg-Arg325 & $38(38.0)$ & $23(8.2)$ \\
\hline ZnT8-Trp-Trp325 & $15(15.0)$ & $9(3.2)$ \\
\hline ZnT8-Arg-Trp325 & $56(56.0)$ & $18(6.4)$ \\
\hline
\end{tabular}

${ }^{a}$ Calculated as percentage of results scored positive by RBA using each variant. 
A
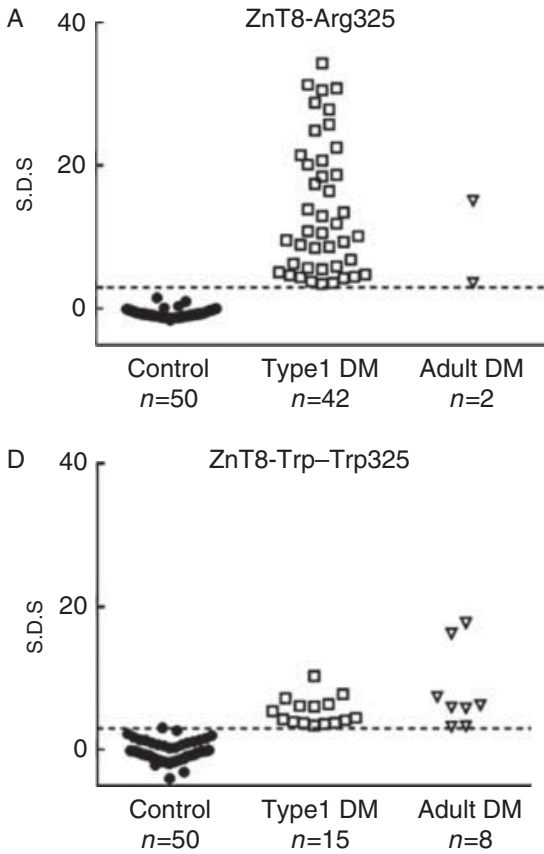
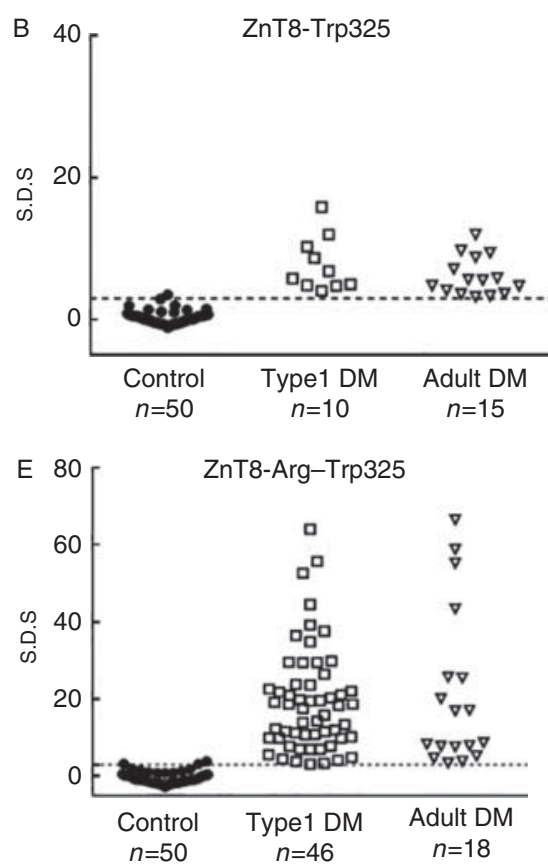

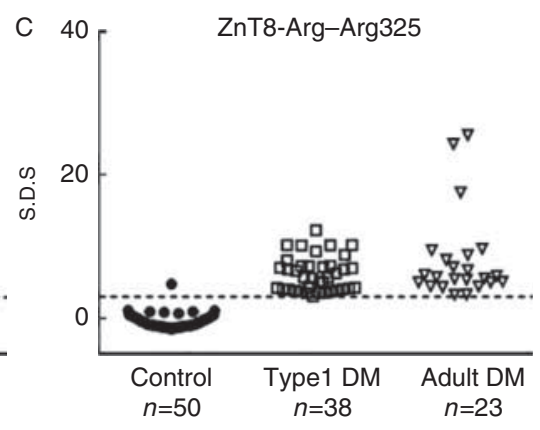

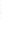

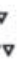

$n=18$

\section{Figure 3}

Signal levels of RBA positive sera to ZnT8-Arg325 (A), ZnT8Trp325 (B), ZnT8-Arg-Arg325 (C), ZnT8-Trp-Trp325 (D), and ZnT8-Arg-Trp325 (E) from type 1 Argentinian diabetic patients

\section{Differential autoantibody reactivities to ZnT8 variants}

Further comparisons were made between the two groups of diabetic patients. Differential reactivity to ZnT8 variants was analyzed and represented in an integrated bar chart (Fig. 4). Out of $65 \mathrm{ZnT} 8 \mathrm{~A}+$ type 1 diabetic patients, 8 recognized all recombinant forms of ZnT8. Most patients (56) reacted against the heterodimer; 25 of them were also positive for the homodimer ZnT8-Arg-Arg325 and monomer ZnT8-Arg325, and only one recognized ZnT8-Trp-Trp325 and ZnT8-Trp325 (Fig. 4A).

In addition, 29 out of $46 \mathrm{ZnT8A}+$ non-obese adultonset diabetic patients recognized only dimers. One patient showed reactivity against ZnT8-Arg325 and ZnT8-Arg-Arg325 exclusively, and six patients were only positive for ZnT8-Trp325 (Fig. 4B).

\section{Discussion}

ZnT8 is a pancreatic $\beta$ cell secretory granule membrane protein that has been recently identified as a target for humoral autoimmunity in type $1 \mathrm{DM}(1,17)$. Unlike GAD and IA-2, ZnT8 is a highly specific $\beta$ cell antigen; therefore, the presence of ZnT8A reveals, although indirectly, and non-obese adult-onset Argentinian diabetic patients. Fifty control subject results were also included for each ZnT8 variant. The cut-off value for each marker is indicated by a dotted line.

a specific damage of these cells. Regarding this new marker, it has been described that $60-80 \%$ of the Caucasian population with type $1 \mathrm{DM}$ and $50-60 \%$ of the Japanese population with acute onset of DM presented ZnT8A (17, 18). In addition, most screening tests for adult-onset autoimmune DM had been based mainly on the detection of GADA, the most prevalent autoantibody, together with the assessment of other markers. Besides, it has been demonstrated that ZnT8A is, in fact, a helpful humoral marker that should be included in diagnostic tests for the screening of Latent Autoimmune Diabetes of

Table 2 Signal levels of ZnT8A positive sera from type 1 DM and non-obese adult onset DM. Data are prsented as S.D.S median.

\begin{tabular}{|c|c|c|}
\hline Antigenic variant & $\begin{array}{c}\text { Type } 1 \text { DM } \\
\text { ZnT8A }+(n=65)\end{array}$ & $\begin{array}{l}\text { Adult onset DM } \\
\text { ZnT8A }+(n=46)\end{array}$ \\
\hline ZnT8-Arg325 & 10.71 & 9.35 \\
\hline ZnT8-Trp325 & 6.32 & 5.61 \\
\hline ZnT8-Arg-Arg325 & 5.66 & 5.78 \\
\hline ZnT8-Trp-Trp325 & 4.44 & 6.07 \\
\hline ZnT8-Arg-Trp325 & $17.99 *$ & 12.87 \\
\hline
\end{tabular}

*Significantly higher than the value for other antigenic variants (Mann-Whitney's $U$ test, $P<0.05$ ). 

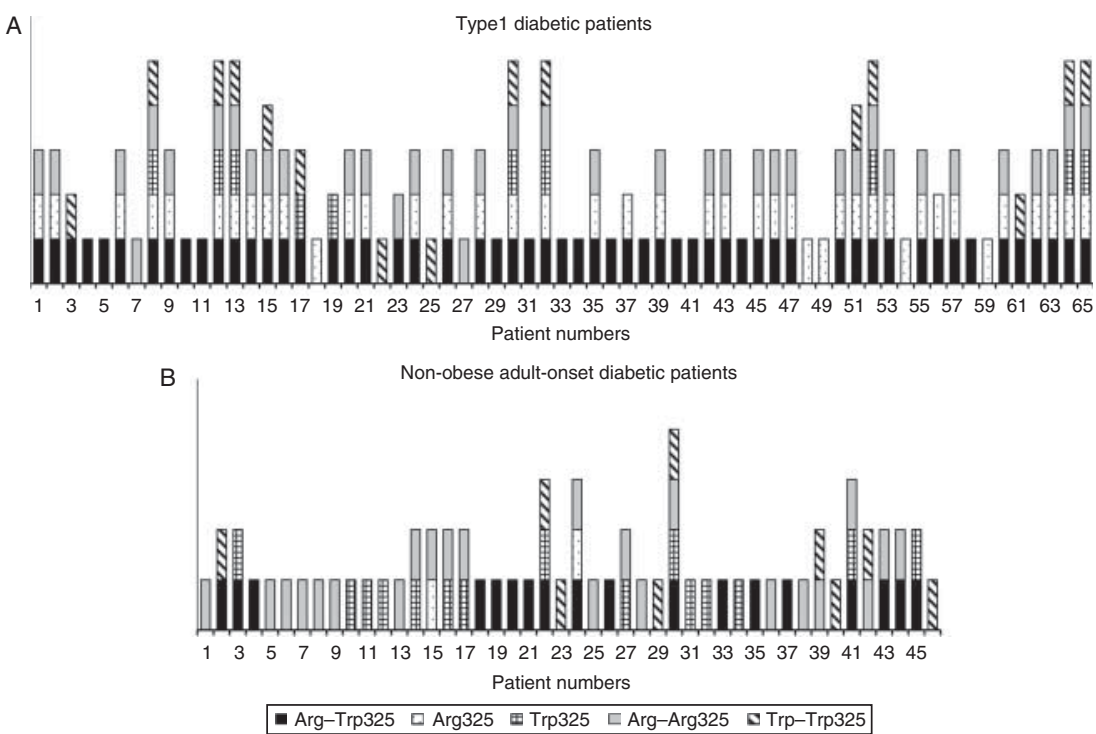

\section{Figure 4}

Autoantibodies differential reactivity to ZnT8 variants in 65 type 1 diabetic patients ZnT8A positive (A) and 46 non-obese adult-onset diabetic patients ZnT8A positive (B).

Adults (LADA). In a previous report $(11,19)$ we have proved that patients with adult-onset DM initially identified as 'marker negative', based on GADA and IA-2A screening, were reclassified as autoantibody positive when tested for ZnT8A. In the present study, we have included homodimer ZnT8 probes in order to improve the differential characterization of ZnT8A in type 1 and non-obese adult-onset diabetic patients.

In this work, we have found that $65 \%$ of patients with recent onset of type $1 \mathrm{DM}$ were $\mathrm{ZnT} 8 \mathrm{~A}+$, confirming the profile described above and increasing the detection of autoimmunity from 84.0 to $93.0 \%$. Moreover, the inclusion of ZnT8A in the screening of autoimmunity in non-obese adult-onset diabetic patients increased the combined sensitivity from 19.9 to 30.1\%. Therefore, ZnT8A constitutes an additional prevalent marker to the preexisting triad (IAA, GADA, and IA-2A), enabling the appropriate classification of diabetic patients as autoimmune DM.

Regarding the epitopes recognized by ZnT8A, it has been described that $80 \%$ of type 1 diabetic patients positive for ZnT8A recognize the ZnT8 C-terminal domain (residues 268-369) with a rare presence of antibodies against the N-terminal fragment (residues 1-74) (20). In this work, we extended the current knowledge of ZnT8A specific recognition from type 1 diabetic patients and non-obese, adultonset diabetic patients using different antigenic variants: monomeric C-terminal domain containing Arg or Trp at residue 325, the heterodimeric construct Arg-Trp proposed by Hutton et al. (12) and the two homodimeric constructions Arg-Arg and Trp-Trp designed in our laboratory. The reason for the employment of such homodimeric chimeras was that Arg or Trp residues in the originally developed heterodimer only attended the presence in a single construct of the two variants due to polymorphisms, whereas the proper contribution of dimerization in the structure of the antigenic determinant was unclear. Hence, we reasoned that the free energy associated with the antibody-antigen interaction must be related mostly to the whole structure of the epitope and only partially in the residue located in the position 325 . Consequently, we speculated that it should be possible to differentiate subpopulations of autoantibodies according to their fine immunoreactivities exhibited by antibodies against the ZnT8 antigenic variants employed. In fact, out of 65 patients with type 1 DM, 25 (38.5\%) presented autoantibodies that recognized only constructions containing Arg. This observation reveals the existence of epitopes dependent of the amino acid present at residue 325, at least partially. These results are in agreement with previous reports published by Kawasaki et al. (10), where the authors concluded that the residue 325 is a key determinant of humoral autoreactivity to ZnT8 and that the SLC3OA8 genotype is important for autoantibody specificity. In fact, we found that ZnT8A positive sera recognizing the monomeric construction with Arg and the three dimeric 
probes (being only negative for the monomer with Trp) confirm not only the existence of epitopes dependent of residue 325 , but also other structural determinants generated by dimerization. This observation could reflect the folding state of $\mathrm{ZnT}$ in vivo.

On the other hand, in non-obese, adult-onset diabetic patients the use of dimers showed the highest percentage of autoimmunity detection. It is reasonable to think that the increase in assay sensitivity may be due to an increased functional avidity of specific antibodies for these best structured antigenic variants.

With the purpose of searching an association between humoral autoreactivity to ZnT8 and SLC3OA8 polymorphism, 115 non-obese, adult-onset diabetic patients were selected for genotyping analysis. Among these patients, 65 (56.5\%) were Arg325 (CC) homozygotes in SLC30A8, 46 (40\%) were CT heterozygotes and 4 (3.5\%) were homozygotes for Trp325 (TT), which was in the Hardy-Weinberg equilibrium $\left(\chi^{2}=1.47, P=0.22\right)$. The minor allele frequency found among our population was 0.235 , similar to the reported allele frequency in Caucasians (0.239 of 226 chromosomes, HapMap project) (21). However, 12 patients were ZnT8A positive, making it not possible to perform a statistical analysis (data not shown).

We conclude that the heterodimeric construction showed higher signal levels and increased dynamic range in RBA for ZnT8A detection. In this sense, it is accepted that the RBA for autoantibodies assessment does not yield a positive result in $100 \%$ of a representative well defined cohort of recent onset type 1 diabetic patients $(22,23)$. The combined screening of other markers, mainly GADA, IA-2A, and IAA/PAA, increases the diagnostic sensitivity; however, about $10 \%$ of the individuals are still negative. The remaining subpopulation of negative patients, may not exhibit other evidences of specific autoimmunity, even though they present permanent insulinopenia and are prone to ketoacidosis. Therefore, these type 1 diabetic patients would be classified as idiopathic (type 1B DM) (14) even when other clinical features and biochemical parameters are usually not in agreement with this hypothesis. An explanation for the suboptimal RBA behavior is that the $\left[{ }^{35} \mathrm{~S}\right]$-tracer synthesized in vitro by a reticulocyte lysate system in native conformation may be not fully representative of the complete or true antigenic entity exposed in vivo during the autoimmune aggression of pancreatic $\beta$ cells and autoantibody induction. In the present work, we have confirmed that the heterodimeric construction showed the best combination of sensitivity and specificity for routine screening of ZnT8A. These observations suggest that conformational non-continuous epitopes arising from the ZnT8 dimeric construction are detected by ZnT8A, emulating the mechanism of immune response triggered by the quaternary structure of this autoantigen in vivo (4). Further studies are necessary to assess the affinity of ZnT8A to dimers and their possible role in the pathogenic process leading to autoinmmune DM.

Finally, we consider that the differential profiles of ZnT8A exhibited by type 1 and non-obese, adult-onset diabetic patients are in line with other observations suggesting the different nature of autoimmune processes underlying both pathologies. In this sense, it has been described that the frequency of the heterozygous $A / G$ genotype in LADA patients is significantly increased compared to type 1 diabetic patients (24). Additionally in previous reports, we have demonstrated different marker profiles (15) and different thermodynamic parameters (affinity and concentration) (25) in both groups of patients. Besides in this work, we observed clinical differences (age at diagnosis and BMI) in both patient groups when their clinical features were correlated with the presence of autoantibodies. As a result, type $1 \mathrm{DM}$ and LADA should be considered as slightly different entities within the 'rainbow' of autoimmune DM (26) and not simple fast and slowly progressive variants of a same pathology.

Supplementary data

This is linked to the online version of the paper at http://dx.doi.org/10.1530/ EJE-15-0681.

\section{Declaration of interest}

The authors declare that there is no conflict of interest that could be perceived as prejudicing the impartiality of the research reported. Informed consent was obtained from all patients for being included in the study.

\section{Funding}

This work was supported in part by grants from FONCYT Program of the National Agency for Science and Technology Promotion (ANPCYT) PICT2008-0998, National Research Council (CONICET) PIP 11220120100415CO, and the University of Buenos Aires, Buenos Aires, Argentina (UBA 20020110100146).

\section{Acknowledgements}

We are grateful to J Hutton from the Barbara Davis Center of Childhood Diabetes, University of Colorado, Aurora, CO, USA, for the gift of CDNA plasmids encoding the C-terminal fragment (amino acids 268-369) of the ZnT8 aa 325-Arg allele and the ZnT8 aa 325-Trp allele. 


\section{References}

1 Wenzlau JM, Juhl K, Yu L, Moua O, Sarkar SA, Gottlieb P, Rewers M, Eisenbarth GS, Jensen J, Davidson HW et al. The cation efflux transporter ZnT8 (Slc30A8) is a major autoantigen in human type 1 diabetes. PNAS 2007104 17040-17045. (doi:10.1073/pnas.0705894104)

2 Chimienti F, Devergnas S, Favier A \& Seve M. Identification and cloning of a $\beta$-cell-specific zinc transporter, ZnT-8, localized into insulin secretory granules. Diabetes 200453 2330-2337. (doi:10.2337/diabetes.53.9.2330)

3 Chimienti F, Devergnas S, Pattou F, Schuit F, Garcia-Cuenca R, Vandewalle B, Kerr-Conte J, Van Lommel L, Grunwald D, Favier A et al. In vivo expression and functional characterization of the zinc transporter ZnT8 in glucose-induced insulin secretion. Journal of Cell Science 2006119 4199-4206. (doi:10.1242/jcs.03164)

4 Murgia C, Devirgiliis C, Mancini E, Donadel G, Zalewski P \& Perozzi G. Diabetes-linked zinc transporter ZnT8 is a homodimeric protein expressed by distinct rodent endocrine cell types in the pancreas and other glands. Nutrition, Metabolism, and Cardiovascular Diseases 200919 431-439. (doi:10.1016/j.numecd.2008.09.004)

5 Pound LD, Sarkar SA, Benninger RK, Wang Y, Suwanichkul A, Shadoan MK, Printz RL, Oeser JK, Lee CE, Piston DW et al. Deletion of the mouse Slc30a8 gene encoding zinc transporter-8 results in impaired insulin secretion. Biochemical Journal 2009421 371-376. (doi:10.1042/ BJ20090530)

6 Nicolson TJ, Bellomo EA, Wijesekara N, Loder MK, Baldwin JM, Gyulkhandanyan AV, Koshkin V, Tarasov AI, Carzaniga R, Kronenberger $\mathrm{K}$ et al. Insulin storage and glucose homeostasis in mice null for the granule zinc transporter ZnT8 and studies of the type 2 diabetes-associated variants. Diabetes 200958 2070-2083. (doi:10.2337/db09-0551)

7 Lemaire K, Ravier MA, Schraenen A, Creemers JW, Van de Plas R, Granvik M, Van Lommel L, Waelkens E, Chimienti F, Rutter GA et al. Insulin crystallization depends on zinc transporter ZnT8 expression, but is not required for normal glucose homeostasis in mice. PNAS 2009 106 14872-14877. (doi:10.1073/pnas.0906587106)

8 Sladek R, Rocheleau G, Rung J, Dina C, Shen L, Serre D, Boutin P, Vincent D, Belisle A, Hadjadj S et al. A genome-wide association study identifies novel risk loci for type 2 diabetes. Nature $2007 \mathbf{4 4 5} 881-885$. (doi:10.1038/nature05616)

9 Wenzlau JM, Moua O, Liu Y, Eisenbarth GS, Hutton JC \& Davidson HW. Identification of a major humoral epitope in Slc30A8 (ZnT8). Annals of the New York Academy of Sciences 20081150 252-255. (doi:10.1196/ annals.1447.028)

10 Kawasaki E, Uga M, Nakamura K, Kuriya G, Satoh T, Fujishima K, Ozaki M, Abiru N, Yamasaki H, Wenzlau JM et al. Association between anti-ZnT8 autoantibody specificities and SLC30A8 Arg325Trp variant in Japanese patients with type 1 diabetes. Diabetologia 200851 2299-2302. (doi:10.1007/s00125-008-1165-y)

11 Trabucchi A, Faccinetti NI, Guerra LL, Puchulu FM, Frechtel GD, Poskus E \& Valdez SN. Detection and characterization of ZnT8 autoantibodies could help to screen latent autoimmune diabetes in adult-onset patients with type 2 phenotype. Autoimmunity $2012 \mathbf{4 5}$ 137-142. (doi:10.3109/08916934.2011.604658)

12 Lampasona V, Schlosser M, Mueller PW, Williams AJ, Wenzlau JM, Hutton JC \& Achenbach P. Diabetes antibody standardization program: first proficiency evaluation of assays for autoantibodies to zinc transporter 8. Clinical Chemistry 201157 1693-1702. (doi:10.1373/ clinchem.2011.170662)
13 Diabetes mellitus. Report of a WHO Study Group. World Health Organization Technical Report Series 1985727 1-113.

14 The Expert Committee of the diagnosis and classification of diabetes mellitus. Report of the Expert Committee on the Diagnosis and Classification of Diabetes Mellitus. Diabetes Care 199720 1183-1197. (doi:10.2337/diacare.20.7.1183)

15 Valdez SN, Sica MP, Labovsky V, Iacono RF, Cardoso AL, Krochik AG, Mazza CS, Ermacora MR, Cedola N \& Poskus E. Combined measurement of diabetes mellitus immunological markers: an assessment of its benefits in adult-onset patients. Autoimmunity 200133 227-236.

16 Valdez SN, Iacono RF, Villalba A, Cardoso Landaburu A, Ermacora MR \& Poskus E. A radioligand-binding assay for detecting antibodies specific for proinsulin and insulin using ${ }^{35}$ S-proinsulin. Journal of Immunological Methods 2003279 173-181. (doi:10.1016/S0022-1759 (03)00197-2)

17 Wenzlau JM, Hutton JC \& Davidson HW. New antigenic targets in type 1 diabetes. Current Opinion in Endocrinology, Diabetes, and Obesity 2008 15 315-320. (doi:10.1097/MED.0b013e328308192b)

18 Kawasaki E, Nakamura K, Kuriya G, Satoh T, Kuwahara H, Kobayashi M, Abiru N, Yamasaki H \& Eguchi K. Autoantibodies to insulin, insulinoma-associated antigen-2, and zinc transporter 8 improve the prediction of early insulin requirement in adult-onset autoimmune diabetes. Journal of Clinical Endocrinology and Metabolism 201095 707-713. (doi:10.1210/jc.2009-1733)

19 Lampasona V, Petrone A, Tiberti C, Capizzi M, Spoletini M, di Pietro S, Songini $\mathrm{M}$, Bonicchio $\mathrm{S}$, Giorgino F, Bonifacio $\mathrm{E}$ et al. Zinc transporter 8 antibodies complement GAD and IA-2 antibodies in the identification and characterization of adult-onset autoimmune diabetes: Non Insulin Requiring Autoimmune Diabetes (NIRAD) 4. Diabetes Care 201033 104-108. (doi:10.2337/dc08-2305)

20 Wenzlau JM, Liu Y, Yu L, Moua O, Fowler KT, Rangasamy S, Walters J, Eisenbarth GS, Davidson HW \& Hutton JC. A common nonsynonymous single nucleotide polymorphism in the SLC30A8 gene determines ZnT8 autoantibody specificity in type 1 diabetes. Diabetes 200857 2693-2697. (doi:10.2337/db08-0522)

21 International HapMap Consortium. The International HapMap Project. Nature 2003426 789-796. (doi:10.1038/nature02168)

22 Bonifacio E, Boitard C, Gleichmann H, Shattock MA, Molenaar JL \& Bottazzo GF. Assessment of precision, concordance, specificity, and sensitivity of islet cell antibody measurement in 41 assays. Diabetologia 199033 731-736. (doi:10.1007/BF00400345)

23 Bingley PJ, Christie MR, Bonifacio E, Bonfanti R, Shattock M, Fonte MT, Bottazzo GF \& Gale EA. Combined analysis of autoantibodies improves prediction of IDDM in islet cell antibody-positive relatives. Diabetes 199443 1304-1310. (doi:10.2337/diab.43.11.1304)

24 Caputo M, Cerrone GE, Lopez AP, Villalba A, Krochik GA, Cedola FN, Targovnik HM \& Frechtel GD. Cytotoxic T lymphocyte antigen 4 heterozygous codon $49 \mathrm{~A} / \mathrm{G}$ dimorphism is associated to latent autoimmune diabetes in adults (LADA). Autoimmunity 200538 277-281. (doi:10.1080/08916930500158203)

25 Trabucchi A, Guerra LL, Faccinetti NI, Iacono RF, Poskus E \& Valdez SN. Surface plasmon resonance reveals a different pattern of proinsulin autoantibodies concentration and affinity in diabetic patients. PLoS ONE 20127 e33574. (doi:10.1371/journal.pone.0033574)

26 Leslie RD, Williams R \& Pozzilli P. Clinical review: Type 1 diabetes and latent autoimmune diabetes in adults: one end of the rainbow. Journal of Clinical Endocrinology and Metabolism 200691 1654-1659. (doi:10.1210/jc.2005-1623)

27 Williams JR. The Declaration of Helsinki and public health. Bulletin of the World Health Organization 200886 650-652. (doi:10.2471/BLT.08.050955)
Received 7 July 2015

Revised version received 9 September 2015

Accepted 13 November 2015 